\title{
Efficient evolutionary algorithm for the thin-film synthesis of inhomogeneous optical coatings
}

\author{
Jinn-Moon Yang and Cheng-Yan Kao
}

\begin{abstract}
We propose an efficient evolutionary approach for the thin-film synthesis of inhomogeneous optical coatings. The proposed approach consists of global and local strategies by integration of decreasingbased mutations and self-adaptive mutations by means of family competition and adaptive rules. $\mathrm{Nu}-$ merical results indicate that the proposed approach performs robustly and is competitive with other approaches. Our approach, although somewhat slower, is flexible and can easily be adopted to other application domains. Our approach is also able to generate homogeneous solutions with two materials available. (C) 2001 Optical Society of America

OCIS codes: $310.1620,310.6860,230.1360$.
\end{abstract}

\section{Introduction}

Optical thin-film coatings are used to change the spectral intensity distribution or the state of polarization of the electromagnetic radiation incident on it for satisfying performance specifications. The coatings have numerous applications, for example, in scientific instrument manufacturing, spectroscopes, medicine, and astronomy. There is a need for many different thin-film design approaches, which are often required for the solutions of different types of problem. These approaches can be roughly divided into analytical, graphical, and numerical methods. ${ }^{1,2}$ Numerical methods, now the most widely used design techniques, formulate the coating design as an optimization problem based on the use of merit functions.

Refinement methods ${ }^{3,4}$ and synthesis methods ${ }^{5,6}$ are two approaches of numerical optical coatings. Refinement methods normally require a starting design, which is often time consuming and difficult to produce in complex systems. A desired solution is then achieved by gradual modification from the starting design. The solution quality of refinement methods is sensitive to the starting point. However, synthesis methods ${ }^{5,7}$ generate their own starting designs. They are usually combined with refinement

J.-M. Yang (moon@csie.ntu.edu.tw) and C.-Y. Kao (cykao@ csie.ntu.edu.tw) are with the Department of Computer Science and Information Engineering, National Taiwan University, Taipei, Taiwan.

Received 7 July 2000.

0003-6935/01/193256-12\$15.00/0

(C) 2001 Optical Society of America methods, because the quality of their solutions is often not good enough. Therefore developing a good synthesis method is an important research topic.

Recently, evolutionary algorithms ${ }^{8,9}$ have been applied to some optical filters and coatings. Evolutionary algorithms are based on ideas borrowed from genetics and natural selection. It is a generally adaptable concept for problem solving, especially well suited for solving difficult optimization problems, in which traditional optimization methods are less efficient. There are roughly three main independently developed but strongly related implementations of evolutionary algorithms: genetic algorithms, ${ }^{10}$ evolution strategies, ${ }^{11}$ and evolutionary programming. ${ }^{12}$ These three types of standard evolutionary algorithm are not efficient. Thus many modifications have been proposed to improve solution quality and to speed up convergence. ${ }^{13}$

In this paper we propose a method called the family competition evolutionary algorithm (FCEA) to synthesize optical thin-film systems with various numbers of layers. The FCEA combines decreasingbased Gaussian mutation, self-adaptive Gaussian mutation, and self-adaptive Cauchy mutation whose performance heavily depends on the same factor, called step size, which is the standard derivation of a Gaussian or a Cauchy distribution. The selfadaptive mutations adapt the step sizes with a stochastic mechanism, and decreasing-based mutations decrease the step sizes with a fixed decreased rate $\gamma$ where $\gamma<1$. To balance exploration and exploitation, these operators are designed to cooperate with one another by incorporation of family competition and adaptive rules to construct the relationship 

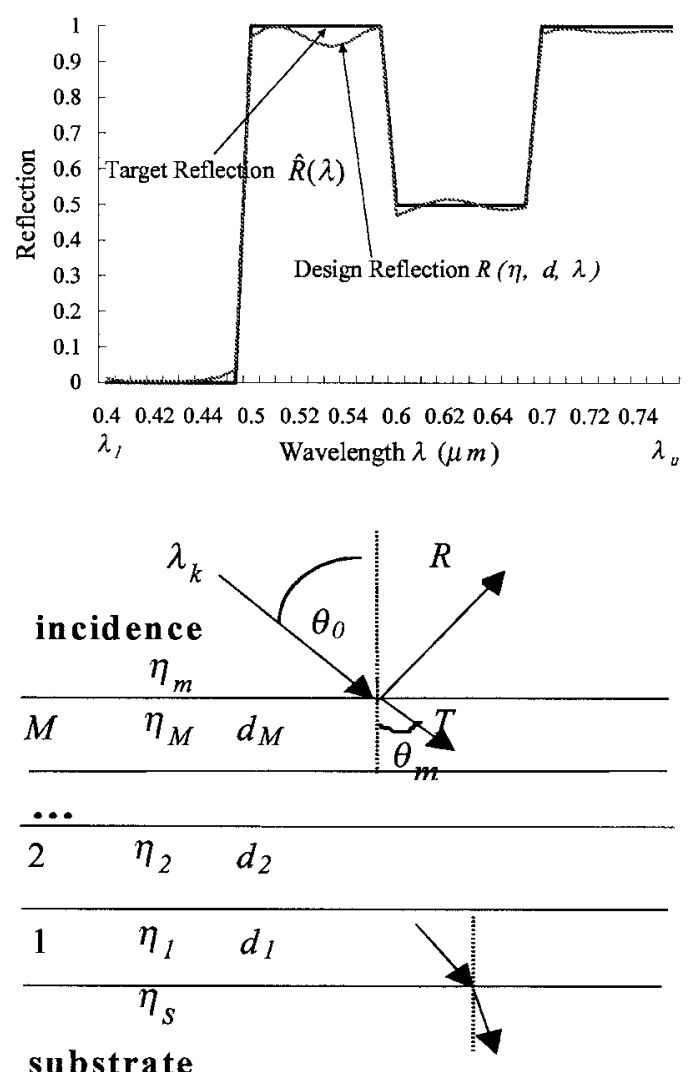

Fig. 1. Top, profiles of a target specification and a real coating system; bottom, construction parameters of a coating system.

among these mutations. Our proposed approach has been successfully applied to homogeneous coatings, ${ }^{14}$ global optimization, ${ }^{15}$ and flexible ligand docking. ${ }^{16}$

The rest of this paper is organized as follows. Section 2 describes the problem of optical thin-film coatings. Section 3 introduces the evolutionary nature of the FCEA. In Section 4 the FCEA is applied to the synthesis of a beam splitter and a narrow-band reflector. The experimental results showed that the FCEA is competitive with the other comparable coating approaches. Furthermore the FCEA synthesized a nonpolarized edge filter that is an oblique-incidence coating. The final problem is the synthesis of a tristimulus filter matching the $\mathrm{CIE}_{\bar{x}_{\bar{x}}}$ used in the tristimulus colorimeters. ${ }^{17}$ Concluding comments are drawn in Section 5.

\section{Problem Definition}

The purpose for the design of optical multilayer coatings is to find the construction parameters of a system that satisfies the desired optical specification. The specification is often is defined by means of specifying the target transmittance $\hat{T}$ or the target reflectance $\hat{R}$ at a number of wavelengths in the spectral region of interest. Figure 1(a) shows examples of a target reflection and a designed reflection. One objective in the designing of a thin-film system is to find the number of layers $M$, the refractive indices $\eta_{j}$, and the thickness $d_{j}$ of the $j$ th layer, in order to match closely with the specified performance where $1 \leq j \leq$ $M$. Figure 1(b) indicates an $M$-layer coating system whose refractive indices are $\eta_{1}, \ldots, \eta_{M}$ and whose thicknesses are $d_{1}, \ldots, d_{M}$.

The merit function a main element of numerical coating methods. Let the spectral reflectance of the $M$-layer system, shown in Fig. 1(b), be denoted as $R(\boldsymbol{\eta}, \mathbf{d}, \lambda)$ where $\lambda$ is the wavelength region of interest. A broadly applicable merit function is given,

$$
f\left(\eta, \mathrm{d}, \lambda_{k}\right)=\left\{\frac{1}{W} \sum_{k=1}^{W} \frac{\left[R\left(\boldsymbol{\eta}, \mathbf{d}, \lambda_{k}\right)-\hat{R}\left(\lambda_{k}\right)\right]^{2}}{\delta R_{k}}\right\}^{1 / 2},
$$

where $R\left(\boldsymbol{\eta}, \mathbf{d}, \lambda_{k}\right)$ and $\hat{R}\left(\lambda_{k}\right)$ are the desired and the target reflectances at the wavelength $\lambda_{k}, \boldsymbol{\eta}$ and $\mathbf{d}$ are the refractive index and the thickness vectors of a coating system, and $\delta R_{k}$ is the tolerance at the wavelength $\lambda_{k}$. In general $\delta R_{k}$ is set to 0.01 . Here $W$ is the number of quantities of interest used in the merit function. The most generally used method of calculating $R\left(\boldsymbol{\eta}, \mathbf{d}, \lambda_{k}\right)$ is based on a matrix formulation. ${ }^{2}$

A coating system is called a normal-incidence coating if the incident angle $\theta_{0}$ shown in Fig. 1(b) is zero, which is otherwise called an oblique-incidence coating. A coating system can be divided into two categories according to the available values of refractive index. It is called an inhomogeneous coating if the values of the refractive-index vector are continuous on the regions of interest; otherwise, it is called a homogeneous coating based on some available materials. The FCEA can be applied to both inhomogeneous and homogeneous coating problems, including the normal- and the oblique-incidence coating problems.

In deposition processes a homogeneous coating system is much easier to construct than an inhomogeneous one. Fortunately, it is relatively simple to convert an inhomogeneous layer to an equivalent two-material layer as long as the thin-layer thickness is small compared with the wavelength of interest. ${ }^{18}$ Of course, the FCEA is also able to generate twomaterial systems directly that consist of only two available materials.

\section{Family Competition Evolutionary Algorithm}

In this section we present the details of the FCEA for the optical thin-film designs. The basic structure of the FCEA is as follows (see Fig. 2): $N$ solutions are randomly generated as the initial population. FCEA then enters the main evolutionary loop, in which each generation consists of three nearly identical procedures. We realize each procedure by performing recombinations, mutations, family competition, and selection. The three procedures differ mainly in the mutations used: decreasing Gaussian mutation $\left(M_{d g}\right)$, self-adaptive Cauchy mutation $\left(M_{c}\right)$, and self-adaptive Gaussian mutation $\left(M_{g}\right)$. Hence we refer to such a procedure as FC_adaptive, which is described below in detail. The output is a new quasi population with $N$ solu- 


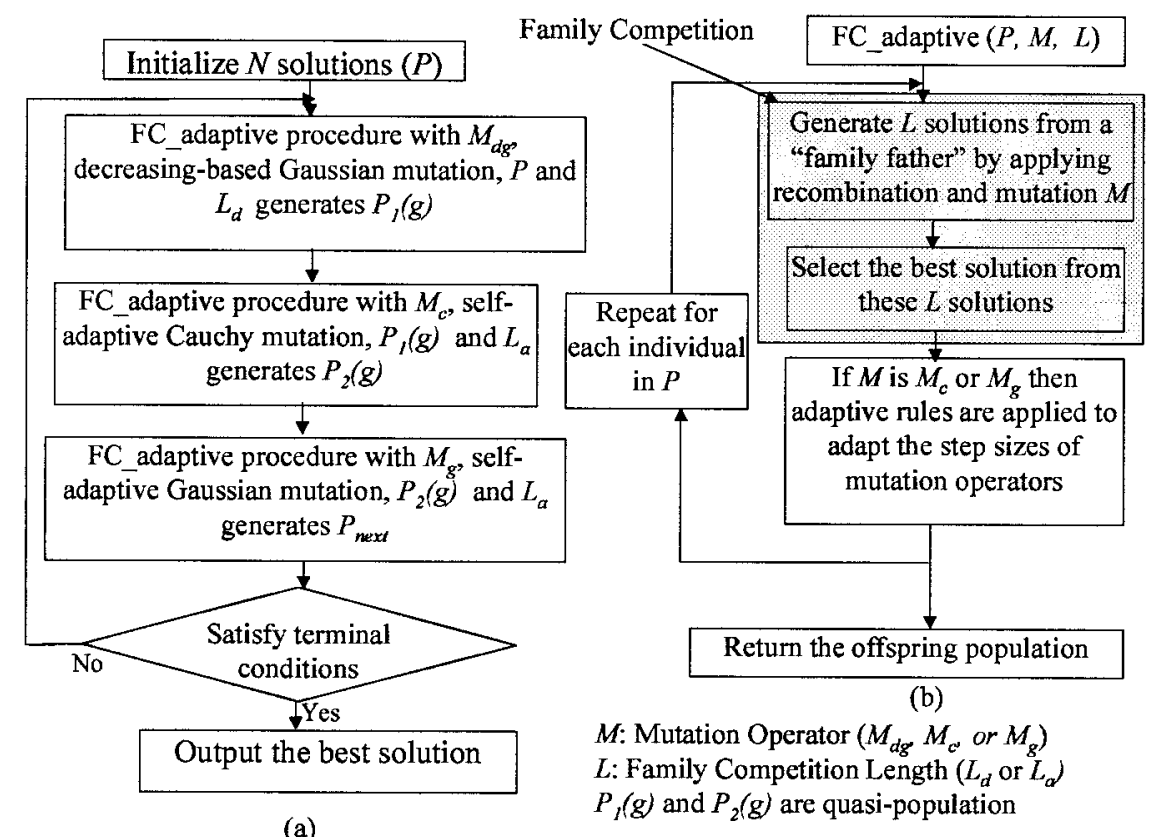

Fig. 2. Overview of our algorithm:

(a) FCEA, (b) FC_adaptive procedure.

tions, which will be the input of the next FC_adaptive procedure.

The FC_adaptive procedure employs three parameters, namely, the parent population $(P$, with $N$ solutions), mutation operator $(M)$, and family competition length $(L)$, to generate a new quasipopulation [Fig. 2(b)]. The FC_adaptive procedure proceeds as follows to generate a quasi population: Each individual in the population sequentially becomes the family father. With a probability $p_{c}$, this family father and another solution randomly chosen from the rest of the parent population are used as parents to perform a recombination operation. Then the new offspring or the family father (if the recombination is not conducted) is operated on by a mutation. For each family father such a procedure is repeated $L$ times. Finally, $L$ children are produced; only that with the best objective value survives. Since we create $L$ children from one family father and perform a selection, this is a family competition strategy. After the family competition, there are $N$ parents and $N$ children left. In each pair of father and child, the individual with a better objective value survives. This is called family selection.

Regarding chromosome representation, we present a solution as $(M, \mathbf{x}, \boldsymbol{\sigma}, \mathbf{v}, \psi)$ in FCEA. $M$ is the number of layers of a coating system. The vector $\mathbf{x}$ includes both the thickness vector and the refractiveindex vector of a coating system to be optimized; $\boldsymbol{\sigma}, \mathbf{v}$, and $\psi$ are the step-size vectors of decreasing-based mutation, self-adaptive Gaussian mutation, and selfadaptive Cauchy mutation, respectively. In other words, each solution $\mathbf{x}$ is associated with some parameters for step-size control. The numbers of elements of the thickness vector and of the refractive- index vector are $M$. Therefore the number of elements of each vector, $\mathbf{x}, \boldsymbol{\sigma}, \mathbf{v}$, and $\psi$, is $2 M$.

In the homogeneous coating, with a pair of materials with $\eta_{l}$ and $\eta_{h}$, the FCEA uses the indicator $I$ to represent the structure of the refractive indices. The refractive index of the first layer is $\eta_{l}$ when $I$ is 0 and is $\eta_{h}$ when $I$ is 1 . The vector $\mathbf{x}$ represents only the thickness vector of a coating system to be optimized. For a two-material system the FCEA represents the chromosome as $(M, I, \mathbf{x}, \boldsymbol{\sigma}, \mathbf{v}, \boldsymbol{\psi})$. In this case the number of elements of each vector, $\mathbf{x}, \boldsymbol{\sigma}, \mathbf{v}$, and $\psi$, is $M$.

The initial value $M$ is randomly chosen from $\left[M_{l}\right.$, $M_{h}$ ] where $M_{l}$ and $M_{h}$ are the numbers of the low- and the upper-bound layers, respectively. The initial value of each entry of $\mathbf{x}$ is randomly chosen over a feasible region depending on the properties of optical coating problems. The initial values for each of the entries of $\boldsymbol{\sigma}, \mathbf{v}$, and $\boldsymbol{\psi}$ are set to $0.04,0.01$, and 0.01 .

For the rest of this section we use inhomogeneous coatings to explain each of the important components of the FC_adaptive procedure-recombination operators, mutation operations, and rules for adapting step sizes $(\boldsymbol{\sigma}, \mathbf{v}$, and $\boldsymbol{\psi})$. To facilitate illustration of the operators, we use $a=\left(M^{a}, \mathbf{x}^{a}, \boldsymbol{\sigma}^{a}, \mathbf{v}^{a}, \boldsymbol{\psi}^{a}\right)$ to represent the family father and $b=\left(M^{b}, \mathbf{x}^{b}, \boldsymbol{\sigma}^{b}, \mathbf{v}^{b}\right.$, $\left.\psi^{b}\right)$ as another parent (only for the recombination operator). The offspring, $c=\left(M^{c}, \mathbf{x}^{c}, \boldsymbol{\sigma}^{c}, \mathbf{v}^{c}, \psi^{c}\right)$, is generated by a genetic operation. We also used the symbol $x_{j}^{d}$ to denote the $j$ th component of an individual $d, \forall j \in\{1, \ldots, 2 M\}$.

\section{A. Recombination Operators}

We have implemented two simple recombination operators to generate offspring: modified discrete recombination and intermediate recombination. With 
probabilities 0.8 and 0.2 , at each FC_adaptive procedure only one of the two operators is chosen. Probabilities are set according to our experimental experience. Here we again note that recombination operators are activated with only a probability $p_{c}$.

\section{Modified Discrete Recombination}

The original discrete recombination generates a child that inherits genes from two parents with equal probability. Here the two parents of the recombination operator are the family father and another solution randomly selected. Our experience indicates that the FCEA can be more robust if the child inherits genes from the family father with a higher probability. Therefore we modified the operator to be as follows:

$$
x_{j}^{c}=\left\{\begin{array}{ll}
x_{j}^{a} & \text { with probability } 0.8 \\
x_{j}^{b} & \text { with probability } 0.2
\end{array} .\right.
$$

For a family father, applying this operator in the family competition is viewed as a local search procedure because this operator is designed to preserve the relationship between a child and its family father.

\section{Intermediate Recombination}

We define intermediate recombination as

$$
\begin{gathered}
x_{j}^{c}=x_{j}^{a}+0.5\left(x_{j}^{b}-x_{j}^{a}\right), \\
w_{j}^{c}=w_{j}^{a}+0.5\left(w_{j}^{b}-w_{j}^{a}\right),
\end{gathered}
$$

where $w$ is $\mathbf{v}, \boldsymbol{\sigma}$, or $\psi$ depending on the mutation operator applied in the family competition. For example, if the self-adaptive Gaussian mutation is used in this FC_adaptive procedure, $\mathbf{x}$ in Eqs. (3) and (4) is v. We emulate the research of the evolution strategies community ${ }^{11}$ to employ only intermediate recombination on step-size vectors, that is, $\boldsymbol{\sigma}, \mathbf{v}$, and $\psi$.

\section{B. Mutation Operators}

Mutations are the main operators of the FCEA. After the recombination, a mutation operator is applied to the family father or the new offspring generated by a recombination. In the FCEA the mutation is performed independently on each vector element of the selected individual by addition of a random value with expectation zero,

$$
x_{i}{ }^{\prime}=x_{i}+w D(\cdot)
$$

where $x_{i}$ is the thickness of the $i$ th of $\mathbf{x}, x_{i}{ }^{\prime}$ is the $i$ th variable of $x^{\prime}$ mutated from $\mathbf{x}, D(\cdot)$ is a random variable, and $w$ is the step size. In this paper $D(\cdot)$ is evaluated as $N(0,1)$ or $C(1)$ if the mutations are, respectively, Gaussian mutation or Cauchy mutation.

\section{Self-Adaptive Gaussian Mutation}

We adapted Schwefel's ${ }^{19}$ proposal to use self-adaptive Gaussian mutation. We accomplish the mutation by first mutating the step size $v_{j}$ and then the thickness $x_{j}$,

$$
\begin{aligned}
& v_{j}^{c}=v_{j}^{a} \exp \left[\tau^{\prime} N(0,1)+\tau N_{j}(0,1)\right], \\
& x_{j}^{c}=x_{j}^{a}+v_{j}^{c} N_{j}(0,1),
\end{aligned}
$$

where $N(0,1)$ is the standard normal distribution. $N_{j}(0,1)$ is a new value with distribution $N(0,1)$ that must be regenerated for each index $j$. For the FCEA we follow Ref. 11 in setting $\tau$ and $\tau^{\prime}$ as $(\sqrt{2 n})^{-1}$ and $\left[(2 \sqrt{n})^{1 / 2}\right]^{-1}$, respectively, where $n=2 M$.

\section{Self-Adaptive Cauchy Mutation}

We follow previous studie ${ }^{20}$ to define self-adaptive Cauchy mutation as follows:

$$
\begin{aligned}
& \psi_{j}^{c}=\psi_{j}^{a} \exp \left[\tau^{\prime} N(0,1)+\tau N_{j}(0,1)\right], \\
& x_{j}^{c}=x_{j}^{a}+\psi_{j}^{c} C_{j}(t) .
\end{aligned}
$$

In our experiments, $t$ is 1 . Note that self-adaptive Cauchy mutation is similar to self-adaptive Gaussian mutation except that Eq. (7) is replaced with Eq. (9). That is, they implement the same step-size control but use different means of updating $\mathbf{x}$.

\section{Decreasing-Based Gaussian Mutations}

Our decreasing-based Gaussian mutation uses the step-size vector $\sigma$ with a fixed decreasing rate $\gamma=$ 0.97 as follows:

$$
\begin{aligned}
& \sigma^{c}=\gamma \sigma^{a}, \\
& x_{j}^{c}=x_{j}^{a}+\sigma^{c} N_{j}(0,1) .
\end{aligned}
$$

Previous results ${ }^{15}$ demonstrated that self-adaptive mutations converge faster than decreasing-based mutations but that, for rugged functions, selfadaptive mutations are more easily trapped into local optima than decreasing-based mutations.

Figure 3 shows the different characteristics of mutation operators of the FCEA. Figure 3(a) compares density functions of Gaussian distribution $[N(0,1)]$ and Cauchy distributions $[C(1)]$. Clearly, Cauchy mutation is able to make a larger perturbation than Gaussian mutation. This implies that Cauchy mutation has a higher probability of escaping from local optima than Gaussian mutation does. For decreasing mutation, the search space for a better child is a hypersphere centered at the parent. However, for self-adaptive mutation, the search space becomes a hyperellipse. Figure 3(b) illustrates this difference by two-dimensional contour plots. Therefore children are searched for in two different types of region.

\section{Adaptive Rules}

The performance of Gaussian and Cauchy mutations is largely influenced by the step sizes. The FCEA adjusts the step sizes while mutations are applied [e.g., Eqs. (6), (8), and (10)]. However, such updates are insufficient for the consideration of the performance of the whole family. Therefore, after family competition, some additional rules are implemented: 


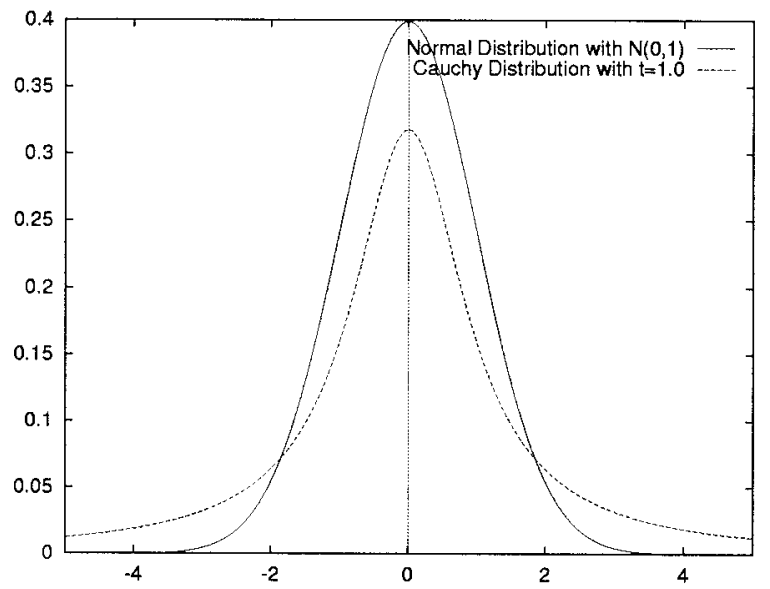

(a)

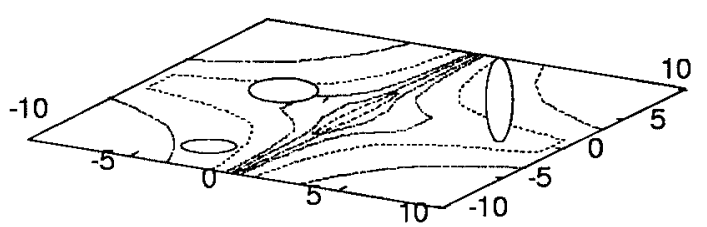

(i)

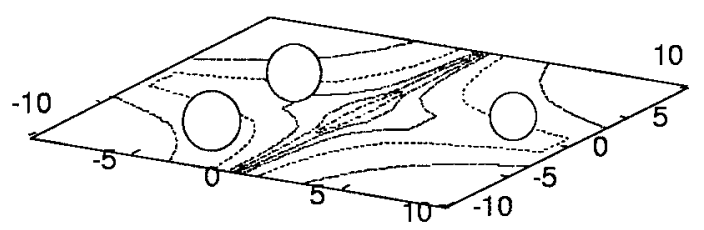

(ii)

(b)

Fig. 3. Different characteristics of FCEA mutation operators. (a) Density functions of Gaussian and Cauchy distributions. (b) Difference in search spaces between (i) self-adaptive and (ii) decreasing-based mutations.

1. A-decrease rule: Immediately after selfadaptive mutations, if objective values of all offspring are greater than or equal to that of the family parent, we decrease the step-size vectors $\mathbf{v}$ (Gaussian) or $\psi$ (Cauchy) of the parent:

$$
w_{j}^{a}=0.95 w_{j}^{a},
$$

where $w^{a}$ is the step-size vector of the parent. In other words, when there is no improvement after self-adaptive mutations, we may propose that a more conservative, that is, smaller, step size tends to make better improvement in the next iteration.

2. D-increase rule: It is difficult, however, to decide the rate $\gamma$ of decreasing mutations. Unlike selfadaptive mutations, which adjust step sizes automatically, the step size of decreasing mutations goes to zero as the number of generations increases. Therefore it is essential to employ a rule that can enlarge the step size in some situations. The step size of the decreasing mutation should not be too small when compared with step sizes of self-adaptive mutations. Here we propose to increase $\sigma$ if one of the two self-adaptive mutations generates better offspring. To be more precise, after a self-adaptive mutation, if the best child with step size $v$ is better than its family father, the step size of the decreasing mutation is updated as follows,

$$
\sigma^{c}=\max \left(\sigma^{c}, \beta v_{\text {mean }}^{c}\right),
$$

where $v_{\text {mean }}{ }^{c}$ is the mean value of the vector $\mathbf{v}$ and $\beta$ is 0.2 in our experiments. Note that this rule is applied in stages of self-adaptive mutations but not of decreasing mutations.

\section{Experimental Results}

In this section we present the numerical results of three inhomogeneous coatings and three homogeneous coatings to illustrate the proposed method. The first and the second problem are used to compare the performance of FCEA with some well-known coating approaches. The third problem, nonpolarized edge filters, and the final problem, $\mathrm{CIE}_{\bar{x}}$ used in the tristimulus colorimeters, are considered to be dif- 
Table 1. Parameters of FCEA and Notation Used in This Paper

\begin{tabular}{ll}
\hline \multicolumn{1}{c}{ Parameter Name } & \multicolumn{1}{c}{ Parameter Value and Notation } \\
\hline Recombination & $p_{c D}=0.8\left(\right.$ for $\left.M_{d g}\right)$ \\
Probability $\left(p_{c}\right)$ & $p_{c A}=0.2\left(\right.$ for $M_{c}$ or $\left.M_{g}\right)$ \\
Family competition & $L_{d}=6\left(\right.$ for $\left.M_{d g}\right)$ \\
Length & $L_{a}=6\left(\right.$ for $M_{c}$ or $\left.M_{g}\right)$ \\
Step sizes & $v_{i}=\psi_{i}=0.01, \sigma_{i}=4 v_{i}$ \\
Population size $(N)$ & 50 \\
Other notation & $M:$ number of layers \\
& $\mathrm{MF}:$ value of merit function \\
& $\sum \eta d$ t total thickness of a solution \\
\hline
\end{tabular}

ficult for refinement methods, because of the lack of good starting points. The experimental results indicate that the FCEA is able to obtain sufficiently good solutions for different types of coating problem.

Table 1 indicates the setting of FCEA parameters, such as initial step sizes, family competition lengths, and recombination probabilities. They are used for the synthesis problems studied in this paper. $L_{d}, \boldsymbol{\sigma}$, and $p_{c D}$ are the parameters for decreasing-based mutation; $L_{a}, \mathbf{v}, \psi$, and $p_{c A}$ are for self-adaptive mutations. The population size is 50. These parameters are decided after experiments have been conducted on these optical coatings with various values. For each problem the FCEA is tested 30 times.

\section{A. Beam Splitter}

We continued from previous research ${ }^{4,8}$ to synthesize a beam splitter in the range of $0.4-1.0 \mu \mathrm{m}$. The target design is the reflectance $R$ specified to $50 \%$ at $0.02-\mu \mathrm{m}$-wavelength increments between 0.4 and 1.0 $\mu \mathrm{m}$; therefore $W$ defined in Eq. (1) is 31 as in previous studies. ${ }^{4,8}$ The incident medium is air $\left(\eta_{m}=1.0\right)$, and the substrate refractive index is glass $\left(\eta_{s}=1.52\right)$. The refractive indices of the layers were allowed to vary freely within the range of 1.35 to 2.35 .

The initial number of layers is randomly chosen from 10 to 20. The initial thickness of each layer was uniformly selected from the region from 0.01 to $0.1 \mu \mathrm{m}$. The FCEA was executed 30 times in all, and the maximum number of generations was 1000 .

The spectral reflectance and the respective refractive-index profile of a solution obtained by the FCEA are shown in Fig. 4. The construction parameters of this solution are given in Table 4, below. The number of layers, the value of the merit function, and the total thickness of this solution are 18, 0.06\%, and $2.0 \mu \mathrm{m}$, respectively. Table 2 shows the comparisons of the FCEA with well-known refinement methods, ${ }^{4}$ such as the dumped-least-square and the Hooke and Jeeves methods, and real-code genetic algorithms ${ }^{8}$ on the beam-splitter filter. The value of the merit function of the best solution obtained by the FCEA is $0.06 \%$. These results show that the FCEA is competitive with these comparable refinement approaches. The FCEA is also more robust than the

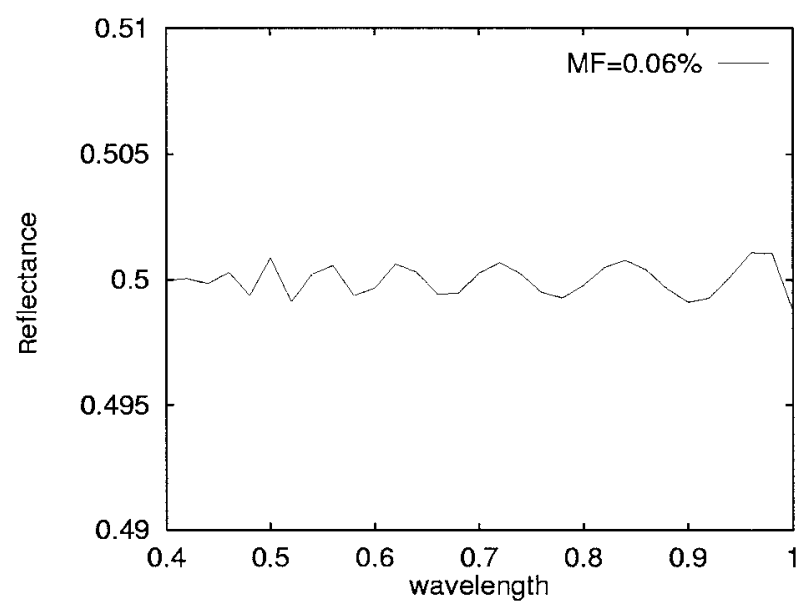

(a) Spectral reflectance

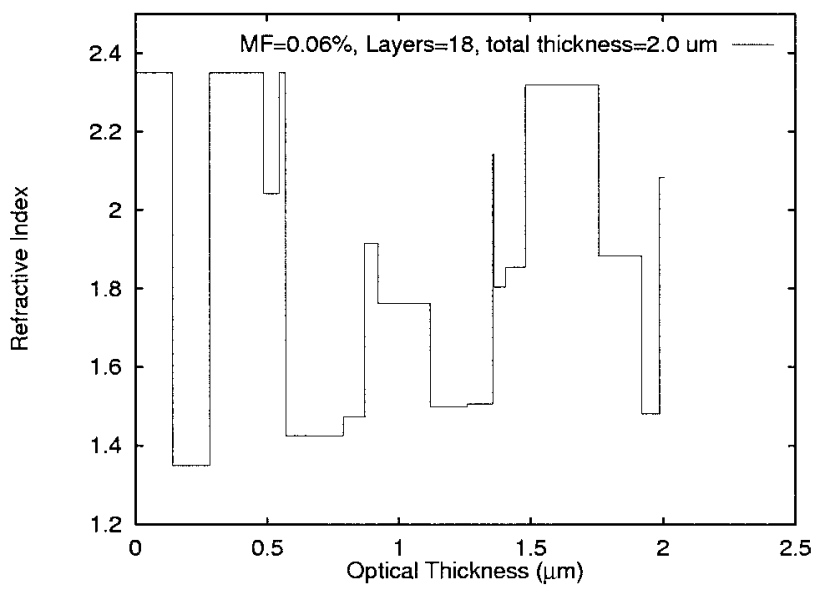

(b) Refractive-index profile (MF $=0.06 \%$ and $\sum \eta d=2.0 \mu \mathrm{m}$ )

Fig. 4. Spectral reflectance and refractive-index profile of an 18layer solution generated by the FCEA for the beam-splitter coating in the region of interest $0.4 \leq \lambda \leq 1.0 \mu \mathrm{m}$ with refractive indices within the range $1.35 \leq \eta \leq 2.35$.

real-code genetic algorithm without the gradient method.

According to the maximum principle, ${ }^{6}$ there is no advantage in using more than two materials each with the lowest $\eta_{l}$ and highest $\eta_{h}$ refractive indices at normal light incidence. That is, the best results can be achieved with a pair of materials that have the lowest and the highest refractive indices. At the same time it is acknowledged that the number of spectral targets can essentially affect the convergence of the optimization routine and the quality of the final result. If this number is insufficient, the convergence and the final results will be poor. Therefore we tested the FCEA on this beam splitter with two materials $\left(\eta_{l}=1.35\right.$ and $\left.\eta_{h}=2.35\right)$ and 301 equispaced points on the wavelength scale, $0.4 \leq \lambda \leq$ $1.0 \mu \mathrm{m}$. The FCEA is also able to obtain good solutions as shown in the homogeneous coating column of Table 4, below, for the beam splitter. The total 
Table 2. Comparisons of FCEA with Refinement Methods ${ }^{a}$

\begin{tabular}{lccccccccc}
\hline & \multicolumn{1}{c}{ FCEA } & $\begin{array}{c}\text { Dumped } \\
\text { Least Square }\end{array}$ & Golden Section & Hooke Jeeves & Rosenbrock & Simplex & RCGA & RCGA + Gradient \\
\hline$M$ & 16 & 18 & 11 & 11 & 11 & 11 & 10 & 20 & 20 \\
$\sum \eta d(\mu \mathrm{m})$ & 1.51 & 2.0 & 1.56 & 1.88 & 1.88 & 1.85 & 1.52 & 2.0 & 2.03 \\
$\mathrm{MF}(\%)$ & 0.097 & 0.06 & 0.28 & 0.97 & 0.37 & 0.50 & 3.81 & 0.41 & 0.06 \\
\hline
\end{tabular}

${ }^{a}$ Methods include those such as dumped least square and golden section and a real-coded genetic algorithm (RCGA) ${ }^{8}$ on the beam splitter with inhomgeneous coating and 31 equispaced points in the region of interest $0.4 \leq \lambda \leq 1.0 \mu \mathrm{m}$. See Ref. 4.

thickness, the number layers, and the value of the merit function of one solution obtained by the FCEA are $1.62 \mu \mathrm{m}, 16$, and $0.0281 \%$, respectively. Figure 5 shows the spectral reflectance and the respective refractive-index profile of this coating system.

In recent years the needle optimization technique has proven to be an extremely efficient optical coating design technique..$^{17,21}$ It is therefore natural to com-

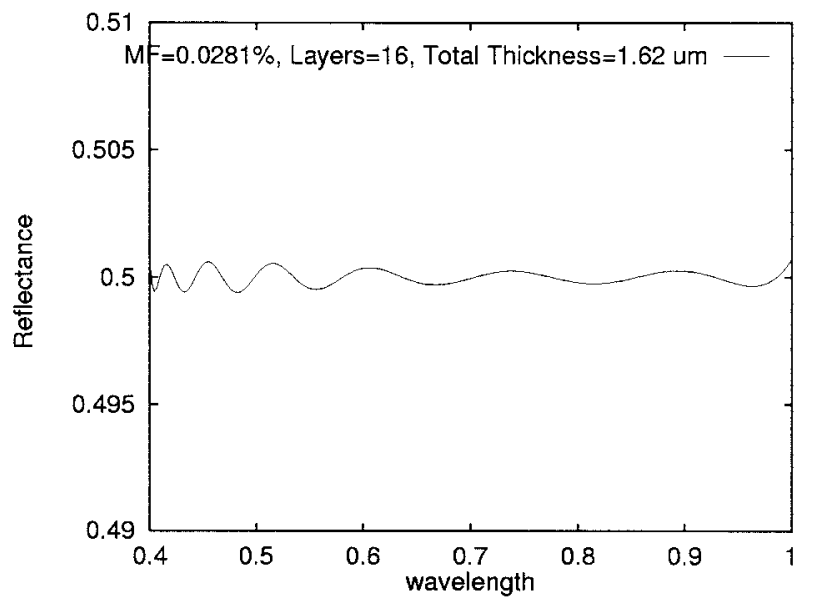

(a) Spectral reflectance

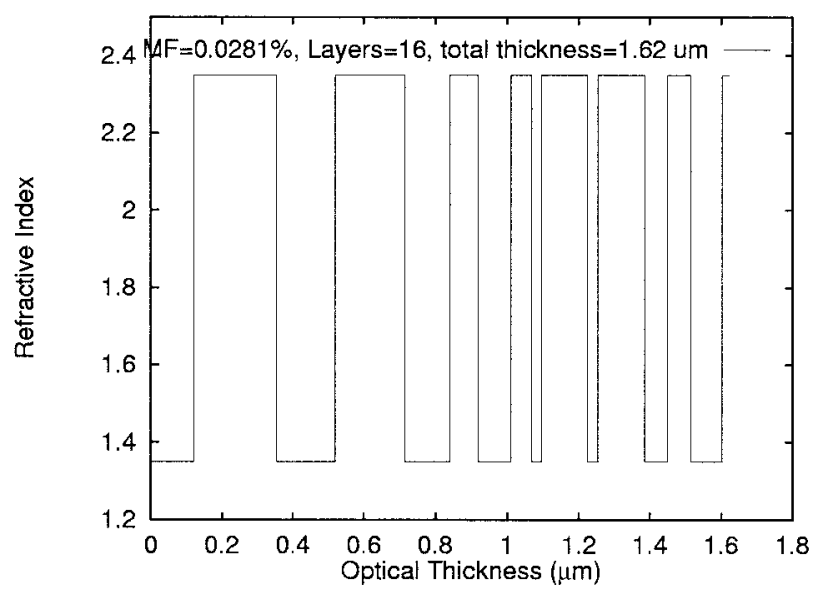

(b) Refractive-index profile (MF $=0.0281 \%$ and $\sum \eta d=$ $1.62 \mu \mathrm{m})$

Fig. 5. Spectral reflectance and refractive-index profile of a 16layer solution generated by the FCEA for the beam-splitter coating with refractive indices of $\eta_{h}=2.35$ and $\eta_{l}=1.35$.
Table 3. Comparison of the FCEA with the Needle Method on the Beam Splitter with Refractive Indices of $\eta_{h}=2.35$ and $\eta_{/}=1.35$ as well as 301 Equispaced Points in the Region of Interest $0.4 \leq \lambda \leq 1.0 \mu \mathrm{m}$

\begin{tabular}{|c|c|c|c|}
\hline Parameter & \multicolumn{2}{|c|}{ FCEA } & Needle Method $^{a}$ \\
\hline Number of layers & 16 & 15 & 14 \\
\hline Total thickness $\left(\sum \eta d\right)(\mu \mathrm{m})$ & 1.62 & 1.65 & 1.51 \\
\hline $\mathrm{MF}(\%)$ & 0.0281 & 0.0311 & 0.0209 \\
\hline
\end{tabular}

${ }^{a}$ Provided by the anonymous referee.

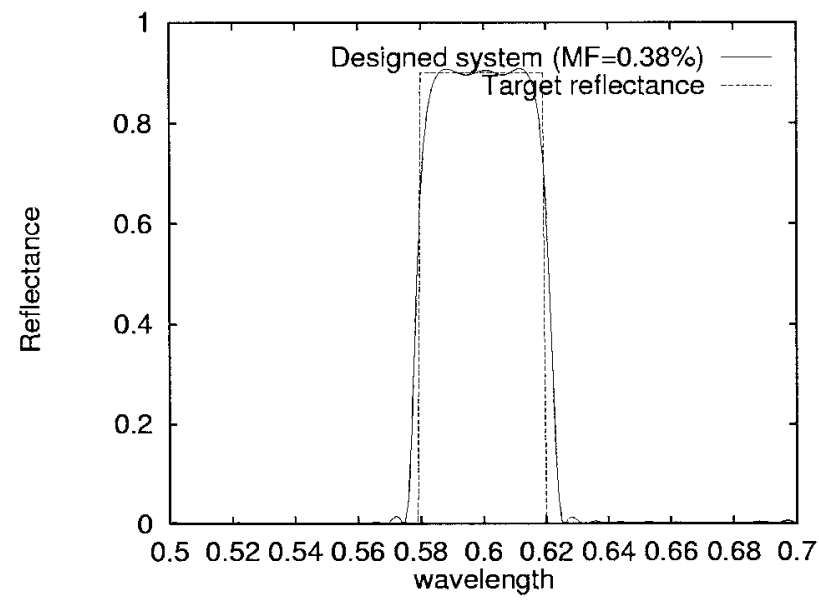

(a) Spectral reflectance

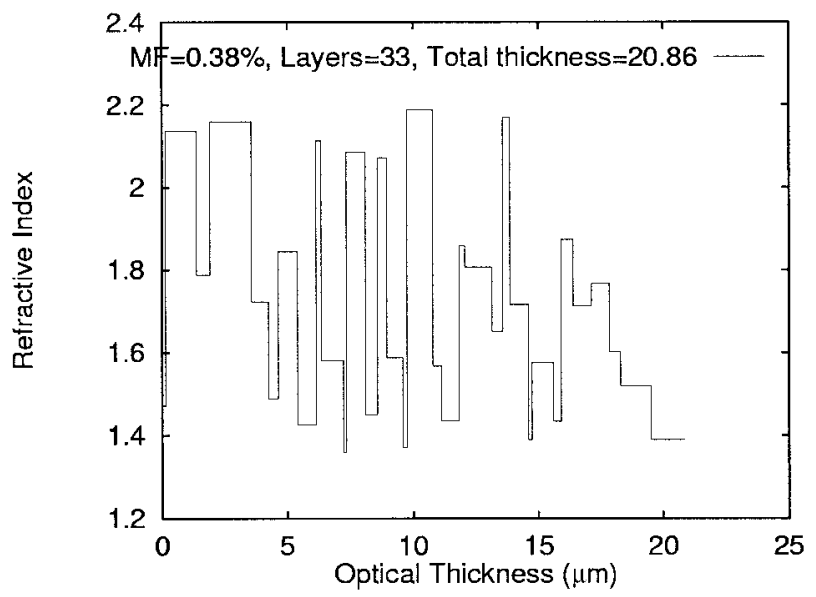

(b) Reflective-index profile

Fig. 6. Spectral reflectance and refractive-index profile of a 33layer narrow-band reflection filter obtained by FCEA in the region of interest $0.5 \leq \lambda \leq 0.7 \mu \mathrm{m}$ on a $\eta_{s}=1.52$ substrate. 
Table 4. Construction Parameters of the Solutions Found by FCEA for the Narrow-Band Reflector, the Beam Splitter, and the $\mathrm{CIE}_{\bar{x}_{\lambda}}$ Filter in Both Inhomogeneous Coatings and Homogeneous Coatings

\begin{tabular}{|c|c|c|c|c|c|c|c|c|c|c|c|}
\hline \multirow[b]{3}{*}{ Layer } & \multicolumn{6}{|c|}{ Beam Splitter } & \multicolumn{4}{|c|}{ Narrow-Band Reflector } & \\
\hline & \multicolumn{2}{|c|}{$\begin{array}{c}\text { Inhomogeneous } \\
\text { Coating }\end{array}$} & \multicolumn{4}{|c|}{$\begin{array}{l}\text { Homogeneous } \\
\text { Coating }\end{array}$} & \multicolumn{2}{|c|}{$\begin{array}{c}\text { Inhomogeneous } \\
\text { Coating }\end{array}$} & \multicolumn{2}{|c|}{$\begin{array}{c}\text { Homogeneous } \\
\text { Coating }\end{array}$} & $\mathrm{CIE}_{\bar{x}_{\lambda}}$ \\
\hline & $\eta$ & $\eta d$ & $\eta$ & $\eta d$ & $\eta$ & $\eta d$ & $\eta$ & $\eta d$ & $\eta$ & $\eta \bar{d}$ & $\eta d$ \\
\hline$\eta_{s}$ & 1.52 & & 1.52 & & 1.52 & & 1.52 & & 1.52 & & 1.52 \\
\hline 1 & 2.0830 & 0.0176 & 2.35 & 0.020105 & 2.35 & 0.0055 & 1.3899 & 1.3361 & 2.35 & 0.2535 & 2.3230 .28000 \\
\hline 2 & 1.4807 & 0.0687 & 1.35 & 0.086979 & 1.35 & 0.0200 & 1.5192 & 1.2387 & 1.35 & 0.2284 & 1.4680 .05073 \\
\hline 3 & 1.8831 & 0.1606 & 2.35 & 0.067596 & 2.35 & 0.0868 & 1.6022 & 0.4550 & 2.35 & 0.2462 & 2.3230 .26493 \\
\hline 4 & 2.3196 & 0.2778 & 1.35 & 0.063868 & 1.35 & 0.0633 & 1.7678 & 0.7085 & 1.35 & 0.1993 & $1.468 \quad 0.14911$ \\
\hline 5 & 1.8542 & 0.0766 & 2.35 & 0.131038 & 2.35 & 0.0607 & 1.7124 & 0.7324 & 2.35 & 0.2046 & 2.3230 .19554 \\
\hline 6 & 1.8031 & 0.0433 & 1.35 & 0.030119 & 1.35 & 0.1116 & 1.8742 & 0.4877 & 1.35 & 0.2301 & 1.4680 .14458 \\
\hline 7 & 2.1438 & 0.0050 & 2.35 & 0.127506 & 2.35 & 0.0130 & 1.4340 & 0.3018 & 2.35 & 0.5720 & 2.3230 .02406 \\
\hline 8 & 1.5058 & 0.0981 & 1.35 & 0.026858 & 1.35 & 0.2826 & 1.5751 & 0.8744 & 1.35 & 0.2490 & 1.4680 .06198 \\
\hline 9 & 1.4988 & 0.1405 & 2.35 & 0.058282 & 2.35 & 0.0610 & 1.3899 & 0.1357 & 2.35 & 0.4155 & 2.3230 .14630 \\
\hline 10 & 1.7621 & 0.1972 & 1.35 & 0.092693 & 1.35 & 0.1130 & 1.7167 & 0.7216 & 1.35 & 0.2534 & 1.4680 .13510 \\
\hline 11 & 1.9163 & 0.0499 & 2.35 & 0.077635 & 2.35 & 0.1076 & 2.1689 & 0.3051 & 2.35 & 0.6011 & 2.3230 .18503 \\
\hline 12 & 1.4736 & 0.0801 & 1.35 & 0.125771 & 1.35 & 0.1999 & 1.6512 & 0.4059 & 1.35 & 0.2300 & $1.468 \quad 0.01468$ \\
\hline 13 & 1.4257 & 0.2186 & 2.35 & 0.194430 & 2.35 & 0.1705 & 1.8065 & 1.1073 & 2.35 & 0.4479 & $2.323 \quad 0.02323$ \\
\hline 14 & 2.3499 & 0.0259 & 1.35 & 0.166489 & 1.35 & 0.2368 & 1.8583 & 0.2180 & 1.35 & 0.2541 & 1.4680 .13814 \\
\hline 15 & 2.0426 & 0.0601 & 2.35 & 0.233750 & 2.35 & 0.1208 & 1.4356 & 0.7107 & 2.35 & 0.2834 & $2.323 \quad 0.28285$ \\
\hline 16 & 2.3500 & 0.2048 & 1.35 & 0.120351 & & & 1.5672 & 0.3301 & 1.35 & 0.2204 & 1.4680 .11565 \\
\hline 17 & 1.3500 & 0.1406 & & & & & 2.1881 & 1.0271 & 2.35 & 1.0605 & 2.3230 .12761 \\
\hline 18 & 2.3499 & 0.1394 & & & & & 1.3711 & 0.1660 & 1.35 & 0.0867 & $1.468 \quad 0.12572$ \\
\hline 19 & & & & & & & 1.5873 & 0.6364 & 2.35 & 0.3216 & 2.3230 .12302 \\
\hline 20 & & & & & & & 2.0727 & 0.3734 & 1.35 & 0.3287 & $1.468 \quad 0.05928$ \\
\hline 21 & & & & & & & 1.4496 & 0.4837 & 2.35 & 0.3072 & 2.3230 .11123 \\
\hline 22 & & & & & & & 2.0855 & 0.7603 & 1.35 & 0.2497 & $1.468 \quad 0.10741$ \\
\hline 23 & & & & & & & 1.3590 & 0.1174 & 2.35 & 0.2010 & $2.323 \quad 0.22387$ \\
\hline 24 & & & & & & & 1.5807 & 0.8820 & 1.35 & 0.2575 & 1.4680 .12111 \\
\hline 25 & & & & & & & 2.1127 & 0.2098 & 2.35 & 0.8326 & 2.3230 .12553 \\
\hline 26 & & & & & & & 1.4254 & 0.7286 & 1.35 & 0.2735 & 1.4680 .05310 \\
\hline 27 & & & & & & & 1.8438 & 0.7653 & 2.35 & 0.5299 & 2.3230 .10685 \\
\hline 28 & & & & & & & 1.4880 & 0.4099 & 1.35 & 0.2313 & 1.4680 .10992 \\
\hline 29 & & & & & & & 1.7227 & 0.6673 & 2.35 & 0.2364 & 2.3230 .06186 \\
\hline 30 & & & & & & & 2.1583 & 1.6515 & 1.35 & 0.2522 & 1.4680 .04284 \\
\hline 31 & & & & & & & 1.7872 & 0.5340 & 2.35 & 0.2796 & $2.323 \quad 0.12914$ \\
\hline 32 & & & & & & & 2.1362 & 1.2458 & 1.35 & 0.3276 & 1.4680 .09496 \\
\hline 33 & & & & & & & 1.4724 & 0.1298 & 2.35 & 0.3110 & 2.3230 .20447 \\
\hline 34 & & & & & & & & & 1.35 & 0.1712 & 1.4680 .01468 \\
\hline 35 & & & & & & & & & & & 2.3230 .09042 \\
\hline 36 & & & & & & & & & & & 1.4680 .01468 \\
\hline 37 & & & & & & & & & & & $2.323 \quad 0.33694$ \\
\hline$\eta_{m}$ & 1.0 & & 1.0 & & 1.0 & & 1.0 & & 1.0 & & 1.52 \\
\hline$\sum \eta d$ & & 2.0 & & 1.62 & & 1.65 & & 20.86 & & 11.14 & 4.597 \\
\hline $\mathrm{MF}(\%)$ & & 0.06 & & 0.0281 & & 0.031 & & 0.381 & & 0.399 & 0.587 \\
\hline
\end{tabular}

pare the FCEA with the needle method. Table 3 shows that the FCEA, although somewhat slower, is comparable with the needle method. The total thickness, the number of layers, and the value of the merit function of a solution found by the needle method are $1.51 \mu \mathrm{m}, 14$, and $0.0209 \%$, respectively. The solution of the needle method is slightly thinner and has fewer layers than those of the FCEA.

\section{B. Narrow-Band Reflection Filter}

The second example concerns the synthesis of a filter in the $0.5-0.7-\mu \mathrm{m}$ region. The substrate and incident medium indices are glass $\left(\eta_{s}=1.52\right)$ and air $\left(\eta_{m}=1.0\right)$, respectively. The target was defined by a reflection equal to $90 \%$ between 0.58 and $0.62 \mu \mathrm{m}$ and zero outside the band in the region of interest. Following previous studies, 7,8 the merit function was defined at 21 points. That is, in each region are seven points. The two edges of the band, between 0.575 and $0.585 \mu \mathrm{m}$ and between 0.615 and 0.625 $\mu \mathrm{m}$, were not specified. The spectral target is the dashed curve as shown in Fig. 6(a) and with the available materials with indices between 1.35 and $2.20 \mu \mathrm{m}$.

The initial number of layers is randomly chosen from 25 to 35 . The initial thickness of each layer was uniformly selected from the $0.01-0.5-\mu \mathrm{m}$ region. The maximum number of generations is 2000 .

Figure 6 shows the spectral reflectance and the refractive-index profile of a final coating system obtained by the FCEA. The number of layers, the value of the merit function, and the total thickness of the solution are $33,0.38 \%$, and $20.86 \mu \mathrm{m}$, respectively. The construction parameters of this solution generated are given Table 4 . The experimental results show that the FCEA is able to obtain coating systems that are close to the target specification. According to the spectral reflections, the FCEA performed better than both the inverse Fouriertransform method $^{7}$ and a real-coded genetic 


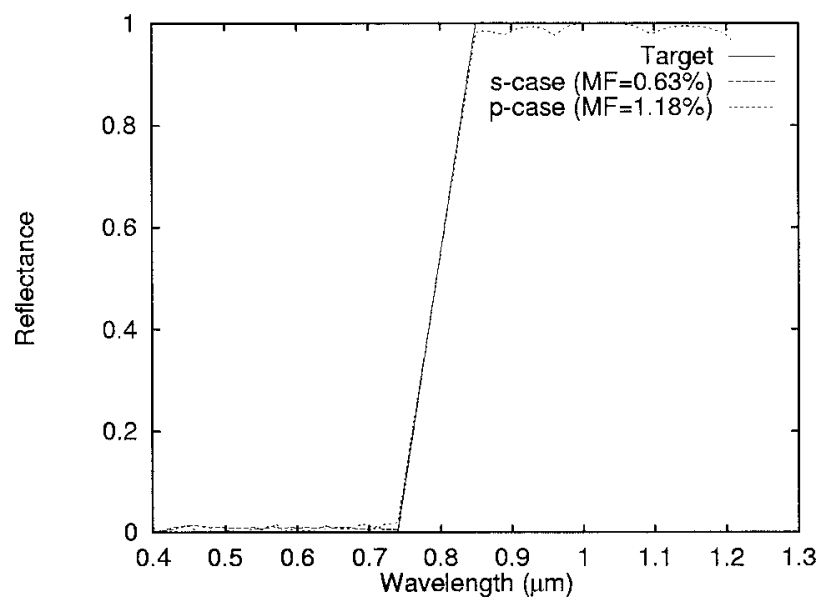

(a) Spectral reflectance

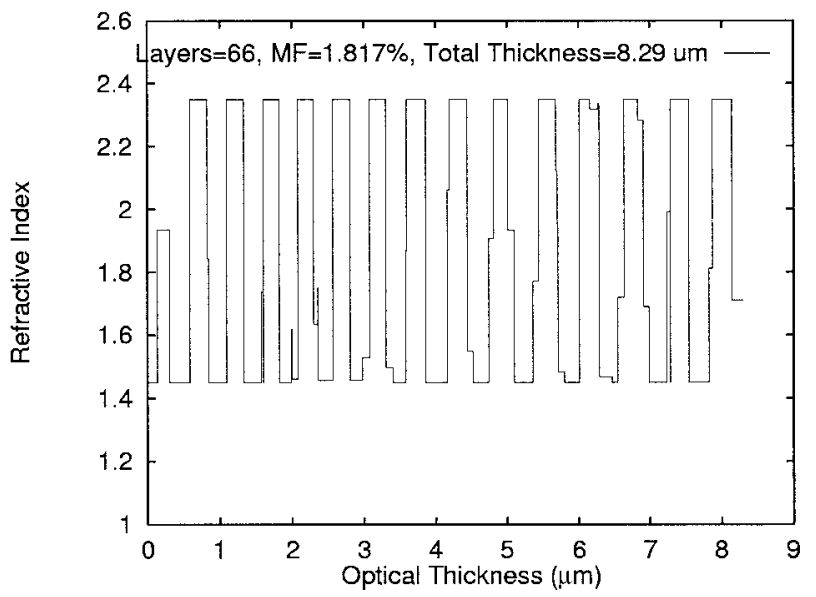

(b) Reflective-index profile

Fig. 7. Spectral reflectance and refractive-index profile of a 66layer short-wave-pass nonpolarized filter obtained by the FCEA at a $45^{\circ}$ angle of incidence on the region of interest $0.4 \leq \lambda \leq 1.2 \mu \mathrm{m}$. The available value of refractive index is continuous from 1.45 to 2.35 .

algorithm. ${ }^{8}$ On comparison, this indicates that the FCEA is closer to the target specification than those obtained by other approaches.

Generally speaking, for thicker designs larger numbers of spectral targets should be used. The solutions of a narrow-band reflection filter are thicker than those of a beam splitter. Thus the FCEA is applied to this filter with two materials $\left(\eta_{l}=1.35\right.$ and $\eta_{h}=2.35$ ) and 213 equispaced points on the wavelength scale, that is, with 71 points existing in each region. In this case the FCEA is able to obtain a good solution; the construction parameters are as shown in the homogeneous coating column of Table 4. Its total thickness is $11.14 \mu \mathrm{m}$, its number of layers is 34 , and its value of the merit function is $0.399 \%$. The average reflectances of this solution are $0.05 \%$ for $0.5 \leq \lambda \leq 0.575,90.31 \%$ for $0.585 \leq \lambda \leq 0.615$, and $0.15 \%$ for $0.625 \leq \lambda \leq 0.7$.

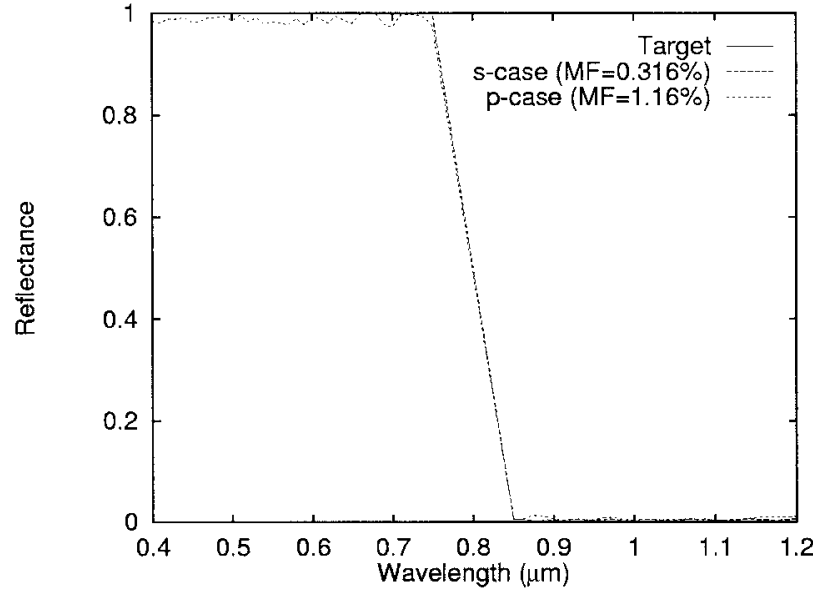

(a) Spectral reflectance

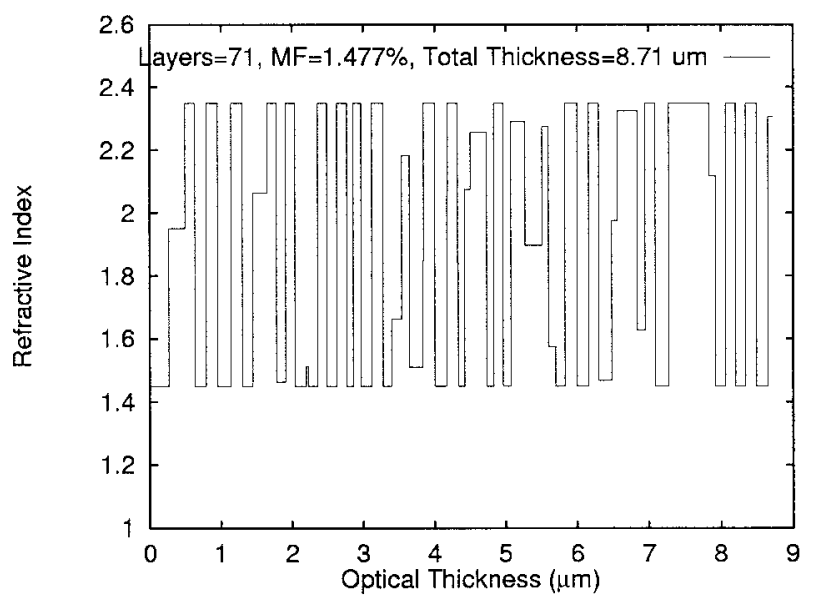

(b) Reflective-index profile

Fig. 8. Spectral reflectance and refractive-index profile of a 71layer long-wave-pass filter for nonpolarized light at a $45^{\circ}$ angle of incidence on the region of interest $0.4 \leq \lambda \leq 1.2 \mu \mathrm{m}$. The refractive index is continuous from 1.45 to 2.35 .

\section{Nonpolarized Edge Filters}

At oblique light incidence the most interesting design is the coating that satisfies for both $s$ and $p$ polarizations simultaneously. In this subsection the FCEA is implemented to design nonpolarization edge filters including the short- and the long-wave-pass filters. The target specifications are that the reflectance is zero in the region from 0.4 to $0.75 \mu \mathrm{m}$ and that the reflectance is 1.0 from 0.85 to $1.2 \mu \mathrm{m}$ for the shortwave-pass filter. For the long-wave-pass filter the reflectance is 1.0 in the region from 0.4 to $0.75 \mu \mathrm{m}$, and the reflectance is zero from 0.85 to $1.2 \mu \mathrm{m}$. The incident angle is $45^{\circ}$, the incident medium is air, and the substrate is glass for these two problems. The merit function was specified at $0.01-\mu \mathrm{m}$-wavelength increments in the region of interest; therefore $W$ defined in Eq. (1) is 72 .

Nonpolarizing coatings are much more difficult to design, and so a large number of layers may be re- 


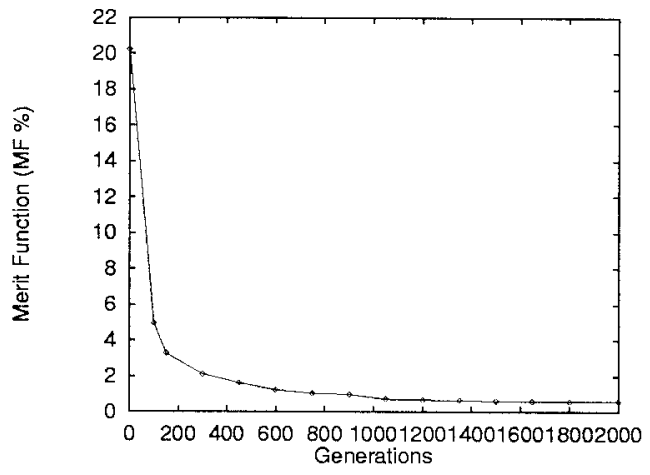

(a) Convergence of MF value

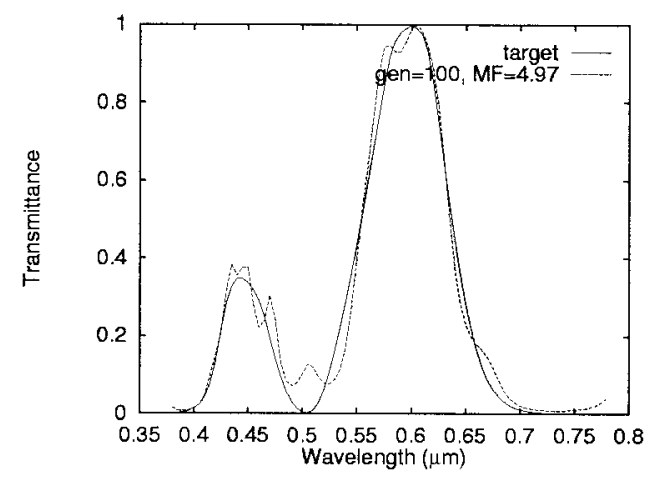

(c) Transmittance (gen $=100$ )

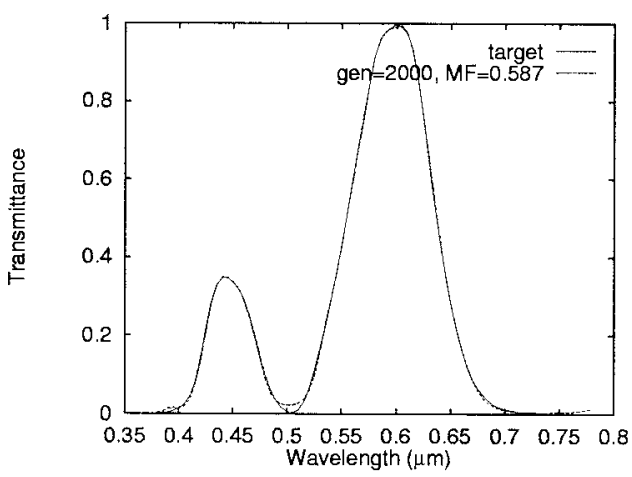

(e) Transmittance (gen $=2000$ )

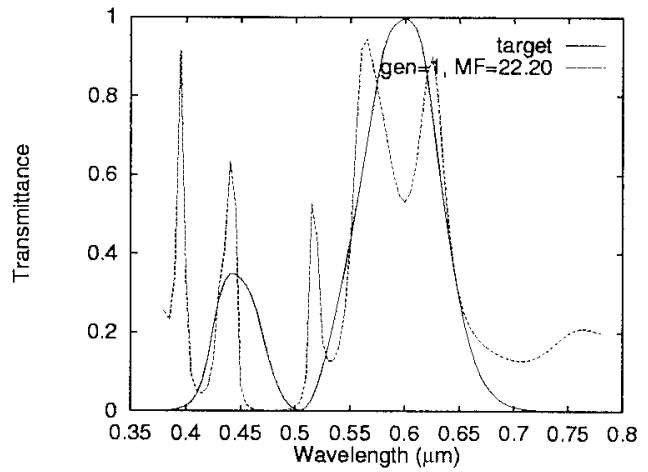

(b) Transmittance (gen=1)

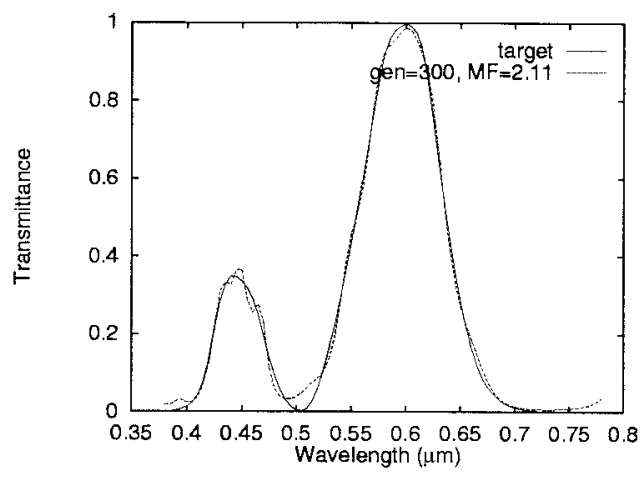

(d) Transmittance (gen $=300$ )

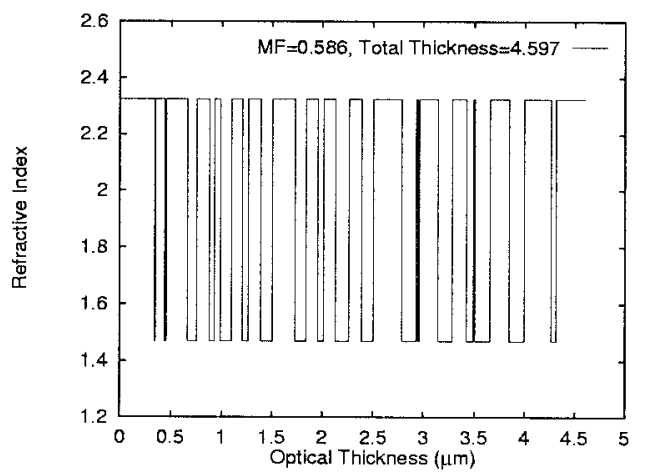

(f) Refractive index (gen $=2000$ )

Fig. 9. Series of intermediate performances and the refractive-index profiles of our FCEA for the CIE $_{\bar{x}_{\lambda}}$ filter for the tristimulus colorimeters in the region $380-780 \mathrm{~nm}$.

quired. Thus the initial number $(M)$ of layers was randomly chosen in the range of 60 to 75 . The initial thickness $\left(d_{j}\right)$ and refractive index $\left(\eta_{j}\right)$ of each layer were uniformly selected from the regions $0.01-0.2$ $\mu \mathrm{m}$ and 1.45-2.35, respectively. The maximum number of generations was set to 2000 .

Figures 7 and 8 show the spectral reflectances and refractive-index profiles of short- and long-wave-pass nonpolarizing edge filters, respectively. The final design of the short-wave-pass filter is a 66-layer coat- ing system, and the value of the merit function is $1.817 \%$. In this final solution, the values of the merit functions of the $s$ - and the $p$-polarized cases are $0.63 \%$ and $1.18 \%$, respectively, as shown in Fig. 7(a) with Fig. 7(b) showing the refractive-index profile.

The long-wave-pass design shown in Fig. 8 has 71 layers with its value of merit function at $1.477 \%$. The values of the merit function of $s$ - and $p$-polarized cases are $0.316 \%$ and $1.161 \%$, respectively. Figure 8(a) shows the $s$ - and the $p$-polarized spectral reflec- 
Table 5. Comparison of FCEA with the Needle Method on the $\mathrm{CIE}_{\overline{\mathbf{x}}_{\lambda}}$ Filter for the Tristimulus Colorimeters in the Region $380-780 \mathrm{~nm}$

\begin{tabular}{|c|c|c|c|}
\hline Parameter & & CEA & Needle Method $^{a}$ \\
\hline Number of layers & 34 & 37 & 31 \\
\hline Total thickness $\left(\sum \eta d\right)(\mu \mathrm{m})$ & 5.13 & 4.597 & 3.982 \\
\hline $\mathrm{MF}(\%)$ & 0.65 & 0.587 & 0.62 \\
\hline
\end{tabular}

${ }^{a}$ Ref. 17.

tances, and Fig. 8(b) shows the refractive-index profile of the solution. These results show that the designs for the $s$ case are easier than those for the $p$ case.

\section{Tristimulus Filter}

In this section we consider the objective of producing a filter that matches the $\mathrm{CIE}_{\bar{x}_{\bar{\lambda}}}$ curve for the standard observer in the 380-780-nm spectral region. ${ }^{17,21}$ This filter is used in the tristimulus colorimeters. The target curve is shown as the solid curve in Fig. 9(b). The final designs should consist of only two coating materials, $\mathrm{SiO}_{2}$ and $\mathrm{Nb}_{2} \mathrm{O}_{5}$, whose refractive indices are $\eta_{l}=1.468$ and $\eta_{h}=2.323$, respectively. Both substrate and medium are assumed to be made of glass whose index is $\eta_{s}=\eta_{m}=1.52$. The merit function was defined at 81 equispaced points on the wavelength scale of interest.

The initial number of layers is randomly chosen from 30 to 40 . The initial thickness of each layer was uniformly selected from the region from 0.01 to $0.1 \mu \mathrm{m}$. The maximum number of generations is 2000 .

Figure 9(a) shows the convergence curve of value of the merit function of the FCEA on the $\mathrm{CIE}_{\bar{x}_{\lambda}}$ filter. Figures 9(b)-Fig. 9(e) illustrate a series of intermediate solutions of a final solution obtained by the FCEA. The dashed curves are the designed results, and the solid curves are the target design. Initially, the value of the merit function is $22.20 \%$. The values of solutions are reduced to $4.97 \%$ and $2.11 \%$ when the numbers of generations approach 100 and 300, respectively. The final solution quality is $0.587 \%$ after the FCEA has exhausted 2000 generations. The number of layers is 37 , and the total thickness is $4.597 \mu \mathrm{m}$ of the final solution. Figure 9(f) shows the refractive-index profile of the final solution. The construction parameters of this solution are also shown in the final column of Table 4.

Table 5 shows that the FCEA is just as good with the needle method ${ }^{17}$ on the $\mathrm{CIE}_{\bar{x}_{\bar{x}}}$ filter. According to the illustrations in Tables 3 and 5, the FCEA is comparable with the needle method, but the solutions of the FCEA are thicker than those of the needle method.

\section{Conclusions}

This study has demonstrated that the FCEA is a stable synthesis approach for optical thin-film designs. Our experience suggests that a global optimization method for synthesizing the optical coating systems should consist of both global and local search strategies. In the FCEA, decreasing-based muta- tions with a large initial step size are a global search strategy; self-adaptive mutations with family competition procedure and replacement selection are local search strategies. These strategies can closely cooperate with each other to improve the overall search performance.

The experiments of four optical coating problems verify that the proposed approach, although somewhat slower, is robust and is competitive with comparable algorithms except for the needle optimization technique, which is an extremely efficient optical coating design method. The FCEA required slightly thick solutions for obtaining almost the same values of the merit function as those of the needle method. The experiments show that the FCEA is able to obtain acceptable solutions whose numbers of layers range from 12 to 85 for different types of coating problem. We believe that the flexibility and robustness of the FCEA make it an effective synthesis method for optical thin-film designs.

We will continue to design a more flexible approach to adapt the number of layers of a coating system and will study a more diverse set of thin-film designs to determine the limits of our FCEA.

\section{References}

1. H. A. Macleod, Thin Film Optical Filters (McGraw-Hill, New York, 1986)

2. J. A. Dobrowolski, "Optical properties of films and coatings," in Handbook of Optics, M. Bass, ed. (McGraw-Hill, New York, 1995), Chap. 42, pp. 2824-2831.

3. J. A. Aguilera, J. Aguilera, P. Baumeister, A. Bloom, D. Coursen, J. A. Dobrowolski, F. T. Goldstein, D. E. Gustafson, and R. A. Kemp, "Antireflection coatings for germanium IR optics: a comparison of numerical design methods," Appl. Opt. 27, 2832-2840 (1988).

4. J. A. Dobrowolski and R. A. Kemp, "Refinement of optical multilayer systems with different optimization procedures," Appl. Opt. 29, 2876-2893 (1990).

5. L. Li and J. A. Dobrowolski, "Computation speeds of different optical thin-film synthesis methods," Appl. Opt. 31, 3790-3799 (1992).

6. A. V. Tikhonravov, "Some theoretical aspects of thin-film optics and their applications," Appl. Opt. 32, 5417-5426 (1993).

7. B. G. Bovard, "Derivation of a matrix describing a rugate dielectric thin film," Appl. Opt. 27, 1998-2005 (1988).

8. S. Martin, J. Rivory, and M. Schoenauer, "Synthesis of optical multilayer systems using genetic algorithms," Appl. Opt. 34, 2247-2254 (1995).

9. H. Greiner, "Robust optical coating design with evolutionary strategies," Appl. Opt. 36, 5477-5482 (1996).

10. D. E. Goldberg, Genetic Algorithms in Search, Optimization and Machine Learning (Addison-Wesley, Reading, Mass., 1989).

11. T. Bäck, F. Hoffmeister, and H.-P. Schwefel, "A survey of evolution strategies," in Proceedings of the Fourth International Conference on Genetic Algorithms (Michigan State University, East Lansing, Mich., 1991), pp. 2-9.

12. D. B. Fogel, Evolutionary Computation: Toward a New Philosophy of Machine Intelligence (Institute of Electrical and Electronics Engineers, Piscataway, N.J., 1995).

13. T. Bäck, U. Hammel, and H.-P. Schwefel, "Evolutionary computation: comments on the history and current state," IEEE Trans. Evol. Comput. 1, 3-17 (1997).

14. J.-M. Yang and C.-Y. Kao, "An evolutionary algorithm for syn- 
thesizing optical thin-film designs," in Parallel Probling Solving in Nature-PPSN V, in Vol. 1498 of Lecture Notes in Computer Science Series (Springer-Verlag, Berlin, 1998), pp. 947-958.

15. J.-M. Yang and C.-Y. Kao, "Integrating adaptive mutations and family competition into genetic algorithms as function optimizer," Soft Comput. 4, 89-102 (2000).

16. J.-M. Yang and C.-Y. Kao, "Flexible ligand docking using a robust evolutionary algorithm," J. Comput. Chem. 21, 988998 (2000).

17. B. T. Sullivan and J. A. Dobrowolski, "Implementation of a numerical needle method for thin-film design," Appl. Opt. 35, 5484-5492 (1996).

18. W. H. Southwell, "Coating design using very thin high- and low-index layers," Appl. Opt. 24, 457-460 (1985).

19. H.-P. Schwefel, Numerical Optimization of Computer Models (Wiley, Chichester, N.Y., 1981).

20. X. Yao and Y. Liu, "Fast evolution strategies," 151-161 (1997).

21. A. V. Tikhonravov, M. K. Trubetskov, and G. W. DeBell, “Application of the needle optimization technique to design of optical coatings,” Appl. Opt. 35, 5493-5508 (1996). 